\title{
Bioinformatics analysis of differentially expressed proteins in alcoholic fatty liver disease treated with recombinant human cytoglobin
}

\author{
ZI-RONG ZHANG $^{1 *}$, ZHENG-GEN YANG $^{2 *}$, YAN-MEI XU ${ }^{1}$, ZHE-YAN WANG $^{1}$, JIAN WEN $^{1}$, \\ BO-HONG CHEN ${ }^{1}$, PING WANG ${ }^{1}$, WEI WEI ${ }^{1}$, ZHEN LI $^{1}$ and WEN-QI DONG ${ }^{1}$
}

${ }^{1}$ Department of Biopharmacy, School of Laboratory Medicine and Biotechnology, Southern Medical University, Guangzhou, Guangdong 510515; ${ }^{2}$ Guangzhou Koncen BioScience Co., Ltd., Guangzhou, Guangdong 510530, P.R. China

Received February 29, 2020; Accepted June 26, 2020

DOI: $10.3892 / \mathrm{mmr} .2021 .11929$

\begin{abstract}
Cytoglobin (Cygb) is a globin molecule that is ubiquitously expressed in all tissues and has a protective role under oxidative stress. It has also been demonstrated to be effective in the treatment of alcoholic fatty liver disease (AFLD). In order to study the molecular mechanisms underlying its beneficial effects for the treatment of alcoholic liver, two-dimensional electrophoresis and mass spectrometric analysis were performed on serum and liver tissues from an in vivo rat model of AFLD. A total of 26 differentially expressed proteins were identified in the serum and 20 differentially expressed proteins were identified in liver specimens. Using online bioinformatics tools, it was indicated that these differentially expressed proteins were primarily associated with pathways including binding and uptake of ligands by scavenger receptors, response to corticosteroid, plasma lipoprotein remodeling, regulation of complement cascade, hydrogen peroxide catabolic process, as well as response to nutrient and monosaccharide. The present results suggested that recombinant human Cygb exerts its role in the treatment of AFLD primarily through affecting nutrient metabolism, monocarboxylic acid biosynthesis, regulation of glutathione expression, plasma lipoprotein remodeling and removal of metabolic waste from the blood.
\end{abstract}

Correspondence to: Dr Wen-Qi Dong or Dr Zhen Li, Department of Biopharmacy, School of Laboratory Medicine and Biotechnology, Southern Medical University, 1838 Guangzhou Avenue North, Guangzhou, Guangdong 510515, P.R. China

E-mail: dongwq63@263.net

E-mail: lizhenlxw@163.com

${ }^{*}$ Contributed equally

Key words: recombinant human cytoglobin, alcoholic fatty liver disease, differentially expressed proteins, molecular mechanism

\section{Introduction}

Alcoholic fatty liver disease (AFLD) is a disease of the liver caused by long-term heavy drinking. Over $90 \%$ of long-term alcoholics suffer from AFLD (1). Without proper treatment, AFLD continues to deteriorate and develops into inflammation, fibrosis and cirrhosis, eventually leading to hepatocellular carcinoma. Fatty liver is usually accompanied by insulin resistance (2), obesity (3) and dyslipidemia (4). AFLD is a worldwide medical problem associated with high morbidity and mortality rates. However, to date, no approved treatments for patients with AFLD are available, and the only strategy for the management of patients with alcoholic liver is to avoid alcohol intake (5). Therefore, the development of novel drugs for the treatment of AFLD is urgently required.

Cytoglobin (Cygb) was first discovered in 2001 (6) and named in 2002 (7). The majority of research indicates that Cygb is a positive regulator of tissue repair and regeneration in multiple organ systems (8). It has been reported that Cygb functions as a peroxidase (6), active oxygen scavenger (9) and nitric oxide plus dioxygenase (10). In animal experiments, recombinant human (rh)Cygb protected hepatic stellate cells from oxidative stress damage and inhibited their differentiation into fibroblasts (11). This has been confirmed by previous experiments in our laboratory, demonstrating that rhCygb improved alcohol-induced liver damage in model rats and significantly reversed serum index elevation (12). Furthermore, it significantly reduced the proliferation of Kupffer cells (KCs) and tumor necrosis factor (TNF)- $\alpha$ expression (12). However, the molecular mechanisms underlying the effects of rhCygb in the treatment of AFLD have remained to be fully elucidated.

In order to explore the underlying molecular mechanisms, a rat model of AFLD was established through 30 weeks of free oral administration of alcohol and the rats were subsequently treated with rhCygb for 10 weeks. The liver and serum samples were extracted to perform 2-dimensional electrophoresis (2-DE) and matrix-assisted laser desorption ionization time of flight (MALDI-TOF) mass spectrometry (MS). Bioinformatics analysis was also performed to predict the possible mechanisms of rhCygb based on the differential 
expression of the proteins. The present study may assist in elucidating the molecular mechanisms of rhCygb and lay a foundation for the development of treatments for AFLD.

\section{Materials and methods}

Animals. Male Wistar rats ( $\mathrm{n}=40$; body weight, $125-155 \mathrm{~g}$; age, 4 weeks) were purchased from the Experimental Animal Center of Southern Medical University. Rats were housed at a constant temperature of $24-25^{\circ} \mathrm{C}$ and $70 \%$ humidity under a controlled 12-h light-dark cycle; they had free access to water and standard rat chow. The experimental protocol followed the guidelines approved by the Chinese Association of Laboratory Animal Care and was approved by the Southern Medical University Animal Care and Use Committee (IACUC).

Induction of AFLD and treatments. Liquor (56\% vol; Beijing Red Star Co., Ltd.), was mixed with distilled water to reach a concentration of $40 \%(\mathrm{v} / \mathrm{v})$. The rats in the alcohol modeling (AM) group $(n=30)$ were administered a liquid diet containing ethanol (liquor) instead of water for 30 weeks. The rats in the negative control (NC) group $(n=10)$ were administered an isocaloric liquid diet containing glucose to replace ethanol. During the 30 weeks, rats had free access to normal food. After 30 weeks, one rat was randomly selected from the $\mathrm{NC}$ group and the AM group and the liver was obtained for pathological sectioning to confirm the formation of AFL. After modeling, the rats in the AM group were randomly divided into the AFLD group $(n=15$; saline $0.5 \mathrm{ml} / \mathrm{kg}$ ) and the rhCygb group $(\mathrm{n}=15 ;$ rhCygb, $3 \mathrm{mg} / \mathrm{kg}$ ). Each group of rats was treated by subcutaneous injection every day for 10 weeks. The animals in the AFLD group and rhCygb group were given alcohol continuously during this period.

Chemical analysis. Blood samples were collected for serum separation as described previously (13). Samples were stored at $-20^{\circ} \mathrm{C}$ for further analysis. Aspartate aminotransferase (AST), alanine aminotransferase (ALT), total cholesterol (T-CHO), triglyceride (TG), high-density lipoprotein cholesterol (HDL-C) and low-density lipoprotein-cholesterol (LDL-C) levels were measured spectrophotometrically using an Olympus AU 5200 (Olympus Corp.).

Pathological evaluation. All rats were anesthetized and sacrificed. The liver tissues were obtained and fixed in $10 \%$ neutral formalin, embedded, cut into $4-\mu \mathrm{m}$ sections and stained with $\mathrm{H} \& \mathrm{E}$ for assessment of steatosis, inflammation and necrosis. Histopathological examinations of the liver sections were performed using an Olympus BX41 image system (Olympus Corp.) as described previously $(14,15)$.

2-DE. Liver samples and serum samples were obtained from the NC group, AFLD group and rhCygb group. A ProteinMiner Protein Enrichment kit (Bio-Rad Laboratories, Inc.) was used to remove high-abundance proteins from serum samples and these were further purified using the 2-D Clean-up kit (Bio-Rad Laboratories, Inc.) for isoelectric focusing (IEF). Serum samples were loaded onto a $17-\mathrm{cm}$ non-linear immobilized $\mathrm{pH}$ gradient (IPG) strip (pH 3-10) and subjected to one-dimensional IEF. The IEF program used was $250 \mathrm{~V}$ for $1 \mathrm{~h}, 500 \mathrm{~V}$ for $1 \mathrm{~h}, 1,000 \mathrm{~V}$ for $1 \mathrm{~h}, 10,000 \mathrm{~V}$ for $3 \mathrm{~h}$ and $500 \mathrm{~V}$ for $1 \mathrm{~h}$.

The liver tissues were thoroughly washed with PBS to remove the effects of serum proteins and the liver samples were frozen in liquid nitrogen and homogenized with a mortar and pestle. Subsequently, the protein samples were treated with protein lysate, nuclease and ultrasound, and finally centrifuged at $40,000 \mathrm{~g}$ and $4^{\circ} \mathrm{C}$ for $1 \mathrm{~h}$ to collect the supernatant. After purification with the 2-D Clean-up kit, liver samples loaded onto a 17-cm linear IPG strip (pH 5-8) for IEF. The IEF program used was the same as that specified above.

After IEF, all of the samples were loaded on a $12.5 \%$ homogeneous SDS gel. The gel was run in parallel at $10 \mathrm{~mA}$ for $60 \mathrm{~min}$, and then at $28 \mathrm{~mA}$ until the bromophenol blue dye reached the bottom. Each experiment was performed in triplicate. The 2-DE gels were stained using the Vorum silver staining method.

Image analysis. A PowerLook 2100XL-USBTM (UMAX) was used for image acquisition, with an optical resolution of 300 DPI and a pixel depth of 8 bits. PDQuest version 8.0.1 (Bio-Rad Laboratories, Inc.) was used for image analysis of 2-DE results. Protein false spots were manually removed and protein classes that were not recognized by the software were added. According to the position, size, shape and other parameters of the spots, the image analysis software automatically matched the same protein spots in different maps and the unmatched protein spots were regarded as the difference points.

In-gel digestion. Gels were eluted with $500 \mu 125 \mathrm{mM}$ ammonium bicarbonate containing 50\% acetonitrile and the supernatant was discarded; this procedure was repeated 3 times, lasting for $60 \mathrm{~min}$ each time. Deposits were eluted once with $500 \mu 1 \mathrm{H}_{2} \mathrm{O}$, the supernatant was discarded and the pellet was dehydrated using $500 \mu \mathrm{l}$ acetonitrile. To break the disulfide bonds of proteins, $10 \mathrm{mM}$ dithiothreitol (DTT) was added for $1 \mathrm{~h}$ and $55 \mathrm{mM}$ iodoacetamide (IAM) was added for alkylation of cysteine in a dark room for $45 \mathrm{~min}$; trypsin solution ( $10 \mathrm{ng} / \mu \mathrm{l}$ in $25 \mathrm{mM}$ ammonium bicarbonate solution) was used to cover it. After $30 \mathrm{~min}$ on ice, excess enzyme solution was removed, and $25 \mu 125 \mathrm{mM}$ ammonium bicarbonate was added to digest deposits overnight at $37^{\circ} \mathrm{C}$. Formic acid (FA; 5\%) was used to terminate the reactions. The samples were eluted with $500 \mu 125 \mathrm{mM}$ ammonium bicarbonate containing $50 \%$ acetonitrile and the supernatant was discarded; this procedure was repeated 3 times, lasting for $60 \mathrm{~min}$ each time. The samples were further eluted once with $500 \mu \mathrm{l}$ of $\mathrm{H}_{2} \mathrm{O}$, the supernatant was discarded and the pellet was dehydrated using $500 \mu \mathrm{l}$ acetonitrile; $10 \mathrm{mM}$ DTT was applied for $1 \mathrm{~h}$ to break the disulfide bonds and subsequently, alkylation of cysteine was performed in a dark room using $55 \mathrm{mM}$ IAM for $45 \mathrm{~min}$. The solution was covered with trypsin solution, left to incubate on ice for $30 \mathrm{~min}$, excess enzyme solution was removed and $25 \mu 125 \mathrm{mM}$ ammonium bicarbonate was added to digest overnight at $37^{\circ} \mathrm{C} .5 \% \mathrm{FA}$ was added to terminate the reaction.

MALDI-TOF-MS data analysis. Peptide mixtures of each gel spot were dissolved in $0.1 \%$ TFA, desalted and concentrated. The samples were then mixed with an equivalent volume of 
matrix ( $\alpha$-cyano-4-hydroxycinnamic acid in $30 \%$ acetonitrile $/ 0.1 \%$ trifluoracetic acid), spotted on a target disk and allowed to air-dry. Samples were analyzed using a Bruker ultrafleXtreme MALDI-TOF MS (Bruker Daltonics). The protein database search was performed using the MASCOT search engine (matrixscience.com; Rat_Uniprot-20190313). Mass tolerance was allowed within 150 parts/million. Proteins matching $>5$ peptides and with a MASCOT score $>60$ were considered significant $(\mathrm{P}<0.05)$.

Bioinformatics analysis. The UNIPROT database (uniprot. org/20190404) was searched for confirming the basic information and function of the protein. To cluster and analyze the function of proteins, metascape (metascape.org/20190515), an online tool, was used for gene ontology (GO) and pathway analysis. In order to clearly illustrate the connection of the clustering network, Cytoscape (version 3.1.2; National Institute of General Medical Sciences of the National Institutes of Health; cytoscape.org) was used to map the analysis results. Briefly, all differentially expressed proteins with enriched GO terms were identified, and accumulative hypergeometric P-values and enrichment factors were calculated and used for filtering. Remaining significant terms were then hierarchically clustered into a tree based on Kappa-statistical similarities among their gene memberships. Subsequently, 0.3 kappa score was applied as the threshold to cast the tree into term clusters. Each term is represented by a circle node, where its size is proportional to the number of input genes fall into that term, and its color represents its cluster identity (nodes of the same color belong to the same cluster). Terms with a similarity score $>0.3$ are linked by an edge (the thickness of the edge represents the similarity score). The network was visualized using Cytoscape (version 3.1.2) with 'force-directed' layout and with edge bundled for clarity. One term from each cluster is selected to have its term description shown as label.

Statistical analysis. Each assay was repeated three times. Values are expressed as the mean \pm standard deviation. The Student's t-test was used to compare differences between two groups. One-way analysis of variance (ANOVA) was used to compare the differences between the vol \% values (fold changes of spot volume) of each identified protein, followed by the least-significant difference (LSD) post-hoc multiple-comparisons test. GraphPad Prism version 5 (GraphPad Software, Inc.) was used for statistical analysis. $\mathrm{P}<0.05$ was considered to indicate a statistically significant difference.

\section{Results}

AFLD treatment with rhCygb. An AFLD rat model was constructed as described previously (12). Compared with those in the NC group, the serum levels of ALT and AST and the AST/ALT ratio were increased in the AM group (Fig. 1A-C). The rat liver of the AM group exhibited notable severe steatosis on H\&E staining (Fig. 1D), indicating that the rats had developed AFLD. After treatment with rhCygb for 10 weeks, compared with that in the AFLD group, the AST/ALT ratio in the rhCygb group was decreased and there was no statistically significant difference between the NC and rhCygb groups (Fig. 1E). In addition, blood lipid levels in each group of rats were analyzed. Compared with those in the NC group, the rats in the AFLD group exhibited abnormal downregulation of HDL-C (Fig. 1F) and abnormal upregulation of TG, T-CHO and LDL-C in the serum (Fig. 1G-I). rhCygb treatment reversed these dyslipidemias and restored the levels of the laboratory indexes to levels similar to those in the NC group (Fig. 1F-I). Fig. 1D displays the complete hepatic lobule structure; there was no fatty degeneration in the NC group, hepatocyte boundaries were clear, the nucleus was located at the center of the cell and hepatocytes were present radially around the central vein. However, in the AM group, the volume of hepatocytes increased, there were lipid vacuoles of different sizes in the cytoplasm, the nucleus of the hepatocytes was squeezed to the side of the cells by vacuoles, the hepatic cord was disorderly arranged and the portal area was accompanied by inflammatory cell infiltration, which was characterized by severe steatosis. The livers of rats treated with rhCygb for 10 weeks displayed a significant reduction in lipid vacuoles and hepatic cords were neatly arranged compared with the AFLD group (Fig. 1J). The results suggested that rhCygb was able to reverse AFLD and dyslipidemias in rats.

2-DE pattern. To analyze the mechanism of the therapeutic effects of rhCygb, protein expression in the serum and liver of animals in the different groups was assessed using 2-DE-based proteomics. The proteins in the three groups had similar patterns, the protein spots were evenly distributed, the proteins from the serum samples were concentrated at the isoelectric point $\mathrm{pH} 3.5-9.5$ and the proteins from the liver samples were concentrated at the isoelectric point $\mathrm{pH}$ 5-8. Based on three repeats, 53 differentially expressed protein spots were obtained in serum samples (Fig. 2) and 40 differentially expressed protein spots in liver samples (Fig. 3).

Protein identification and bioinformatics analysis. The gel spots of differentially expressed proteins were excised and identified by MALDI-TOF-MS. By using the MASCOT search engine to query the NCBInr protein database, 26 proteins in the serum samples were determined to be differentially expressed with confidence (Table I) and 20 proteins in the liver samples with confidence (Table II). A comparative analysis of the expression levels of each proteins in the tables between different groups was conducted. Comparing the AFLD group with the NC group indicated that there were abnormal expression level changes of these proteins in the AFLD group. Comparing the rhCygb group with the AFLD group suggested whether rhCygb could reverse the abnormal changes of these proteins.

GO enrichment and Reactome Gene Sets analysis of the 26 differentially expressed proteins in the serum samples was performed and 7 classifications were obtained: Binding and uptake of ligands by scavenger receptors, response to corticosteroid, plasma lipoprotein remodeling, regulation of the complement cascade, blood circulation, acute inflammatory response and camera-type eye development (Fig. 4A). Table III presents the gene symbols corresponding to the proteins contained in the $\mathrm{GO}$ enrichment analysis. GO enrichment analysis and Reactome Gene Sets of the 20 differentially expressed proteins from the liver samples indicated 5 classifications: Hydrogen peroxide catabolic process, response 

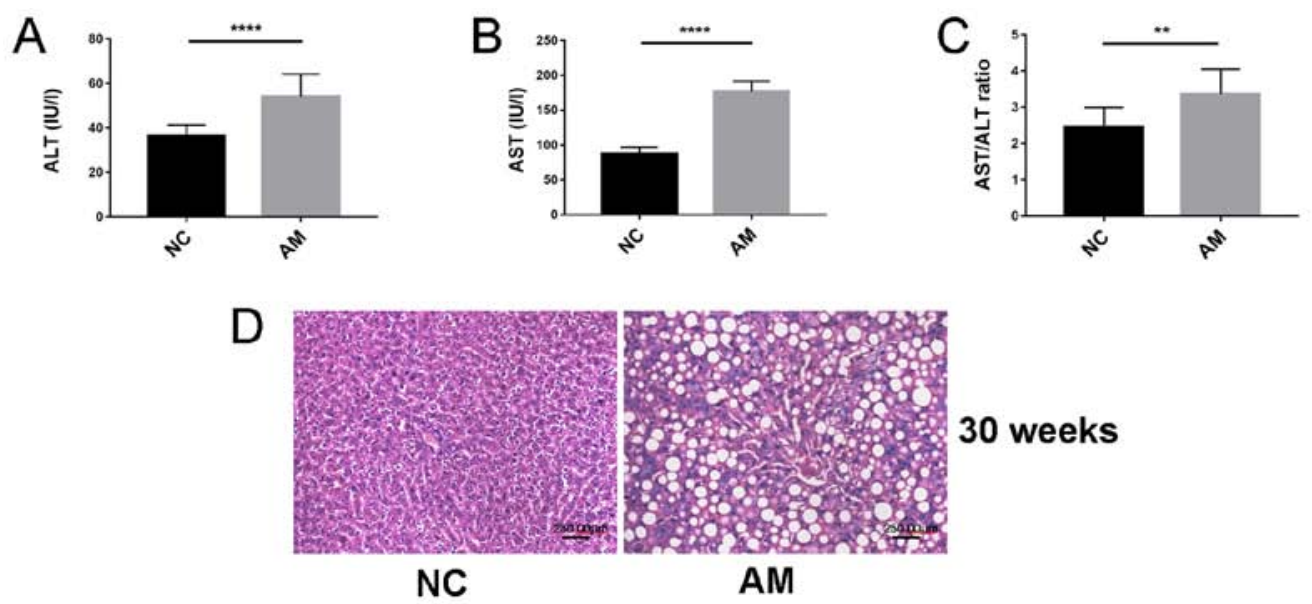

\section{0 weeks}
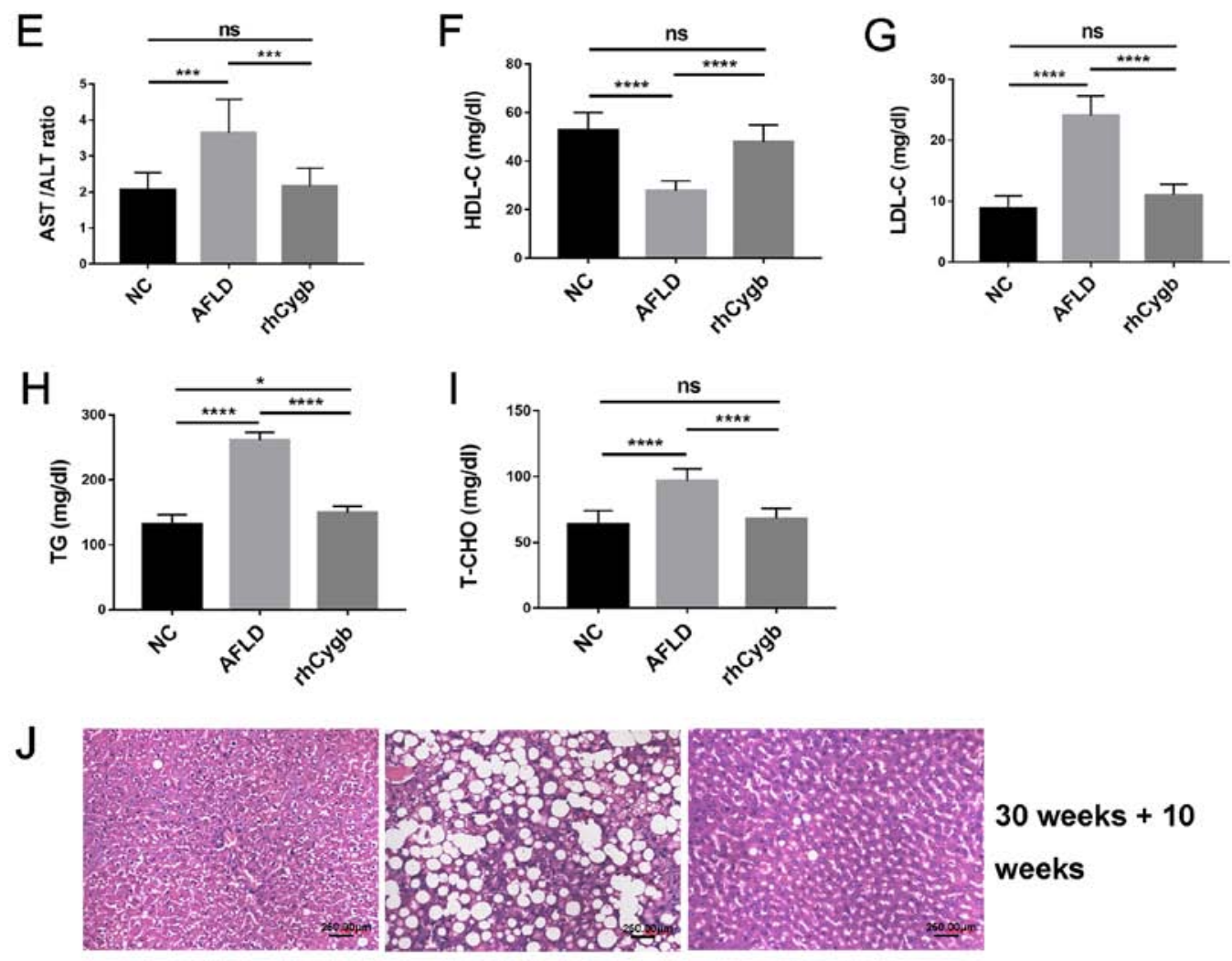

NC

AFLD

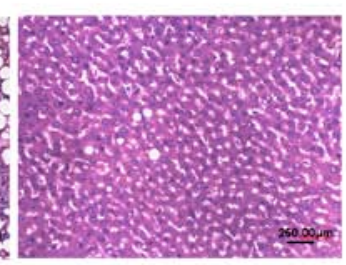

30 weeks +10

weeks

Figure 1. Effect of rhCygb on histology and serum biomarkers in rats with AFLD. ALT levels in the NC and AM groups (A) prior to and (B) after treatment. (C) Comparison of the AST/ALT ratio in the NC group and AM group prior to treatment. (D) Representative images for the NC group and AM group after 30 weeks of modeling. (E) Comparison of the AST/ALT ratio in the NC group, AFLD group and rhCygb group after treatment. (F) HDL-C, (G) LDL-C, (H) TG and (I) T-CHO values of the NC, AFLD and rhCygb groups after treatment. (D and J) Representative images of H\&E staining to observe the morphology of livers from the different groups (magnification, x200). (J) Representative images for the NC group, AFLD group and rhCygb group after 30 weeks of modeling followed by 10 weeks of treatment (scale bar, $250 \mu \mathrm{M})$. Values are expressed as the mean \pm standard deviation $(\mathrm{n}=3)$. ${ }^{*} \mathrm{P}<0.05$, ${ }^{* *} \mathrm{P}<0.01 ;$ ${ }^{* * *} \mathrm{P}<0.001 ;{ }^{* * * * *} \mathrm{P}<0.0001$. ns, no significance; AST, aspartate aminotransferase; ALT, alanine aminotransferase; TG, triglycerides; T-CHO, total cholesterol; HDL-C, high-density lipoprotein cholesterol; LDL-C, low-density lipoprotein cholesterol; NC, negative control; rhCygb, recombinant human cytoglobin; AFLD, alcoholic fatty liver disease; AM, AFLD model.

to nutrient, response to monosaccharide, monocarboxylic acid biosynthetic process, and response to peptide hormone (Fig. 4B); Table IV shows the gene symbols corresponding to the proteins contained in the $\mathrm{GO}$ enrichment analysis.

Metascape was used to perform statistically terminological enrichment of the 12 upregulated proteins and 14 downregulated proteins in the serum proteins and the 14 upregulated proteins and 6 downregulated proteins in the liver samples of rats treated with rhCygb. A subset of representative terms was selected from the enriched terms and converted to a network layout. For the serum samples, the terms contained in the network included binding and uptake of ligands by scavenger receptors, response to corticosteroid, plasma lipoprotein remodeling, regulation of complement cascade, blood circulation, acute inflammatory response and camera-type eye development (Fig. 4C). For the liver samples, the terms 

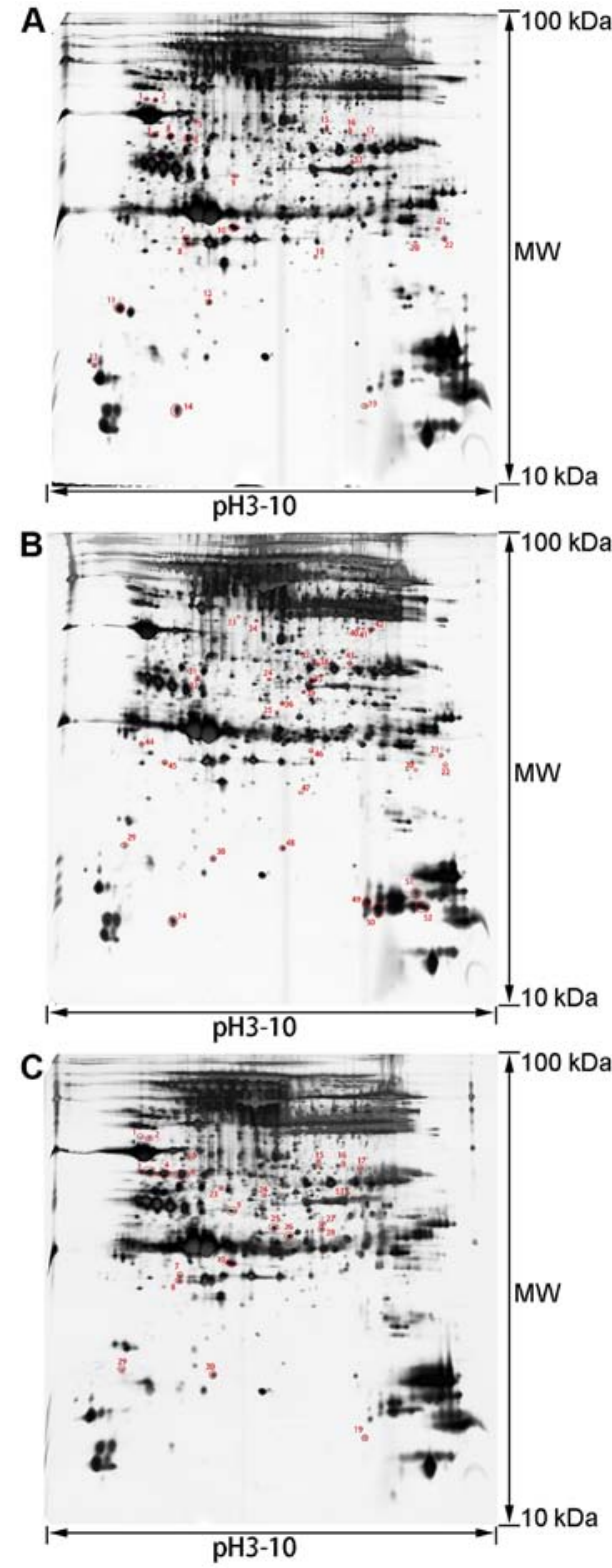

Figure 2. Two-dimensional electrophoresis analysis of serum samples (A) Negative control group. (B) Alcoholic fatty liver disease group. (C) Recombinant human cytoglobin group. Using a pH 3-10, 17-cm nonlinear immobilized $\mathrm{pH}$ gradient strip and $12.5 \%$ SDS-PAGE, 53 different proteins were isolated from the gels. MW, molecular weight.

contained in the network included hydrogen peroxide catabolic process, response to nutrient, response to monosaccharide, monocarboxylic acid biosynthetic process and response to peptide hormone (Fig. 4D).

In the visualization network of the serum samples (Fig. 4C), rhCygb may have exhibited its effects primarily via two parts: i) The purple cluster represented by regulation of complement cascade, which was notably distinct from the other six clusters; and ii) the remaining six clusters (not including the purple cluster) consisted of red cluster represented by binding and uptake of ligands by scavenger receptor, a blue cluster represented by response to corticosteroid, green cluster represented by plasma lipoprotein remodeling, orange cluster represented by blood circulation, yellow cluster represented by acute inflammatory response and a brown cluster represented
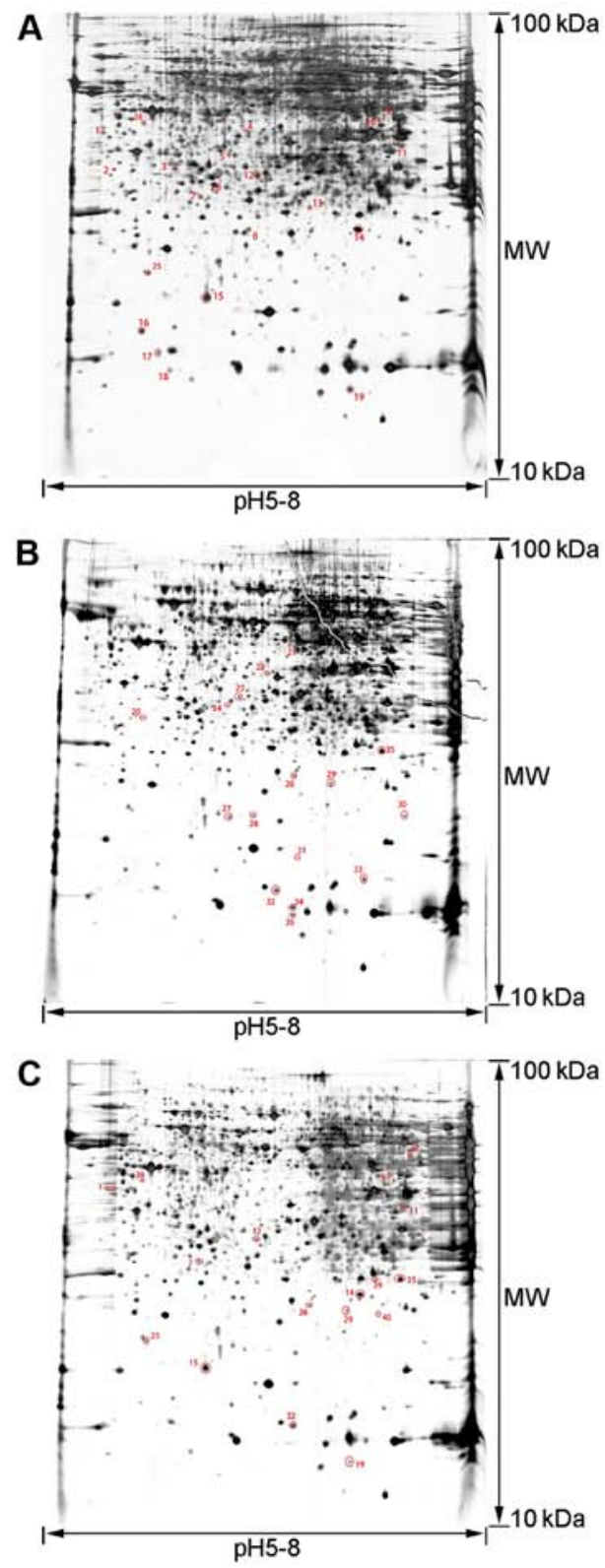

Figure 3. Two-dimensional electrophoresis analysis of liver samples. (A) Negative control group. (B) Alcoholic fatty liver disease group. (C) Recombinant human cytoglobin group. Using a pH 5-8, 17-cm linear immobilized $\mathrm{pH}$ gradient strip and $12.5 \%$ SDS-PAGE, 40 different proteins were isolated from the gels. MW, molecular weight.

by camera-type eye development. In the six clusters on the right, three groups of blue, green and orange were more closely associated with each other and even intertwined (the thickness of the edge represents the similarity score), indicating that rhCygb may be capable of affecting metabolites in serum. In the visualization network of liver samples (Fig. 4D), red term clusters represented by hydrogen peroxide catabolic process, blue term clusters represented by response to nutrient, green term clusters represented by response to monosaccharide and orange term clusters represented by response to peptide hormone were associated with each other (these three clusters linked with each other and had wide edge), where red term clusters were most closely associated with green term clusters (the purple link is the darkest). Blue term clusters and orange term clusters were less closely associated with other term 


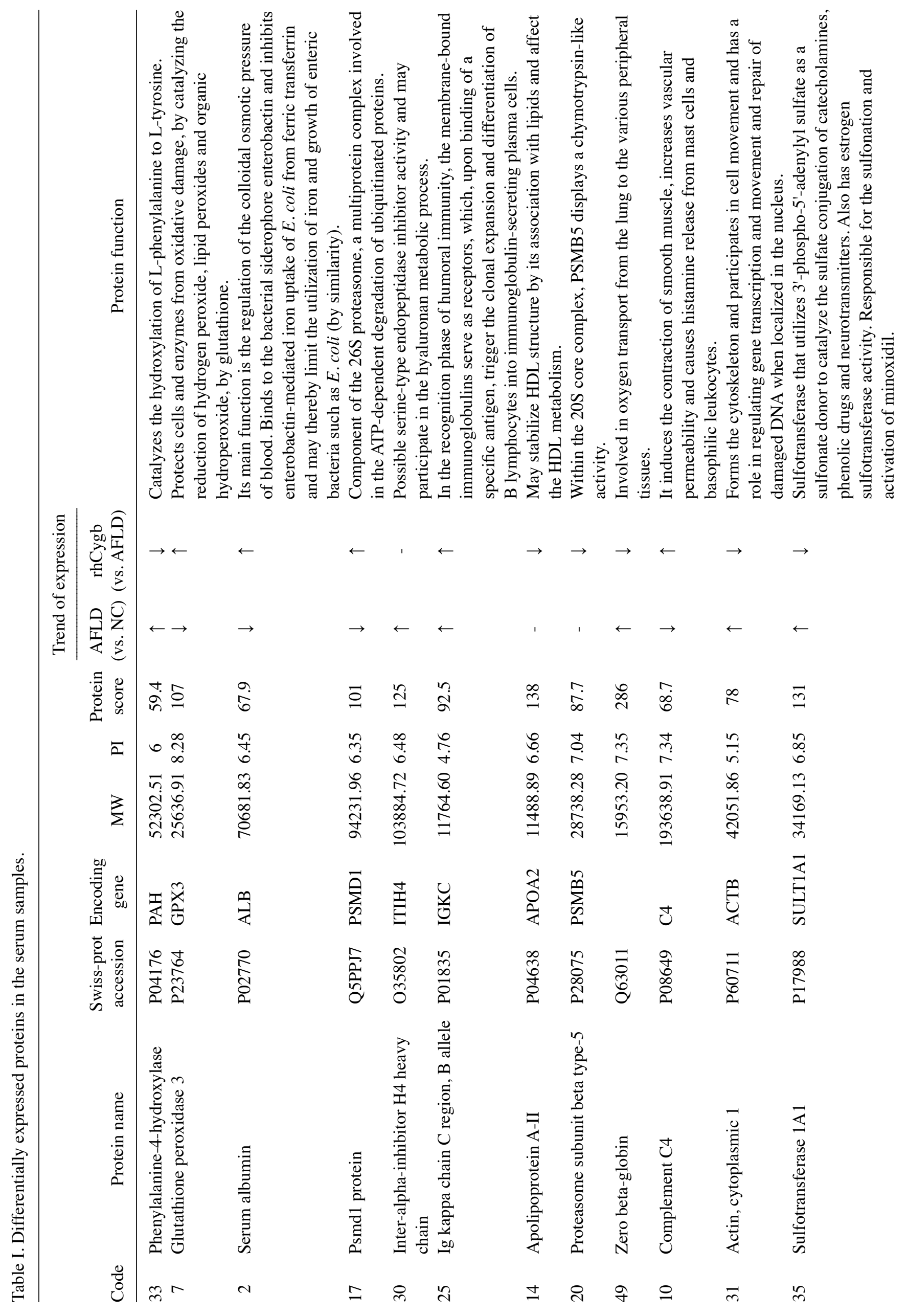




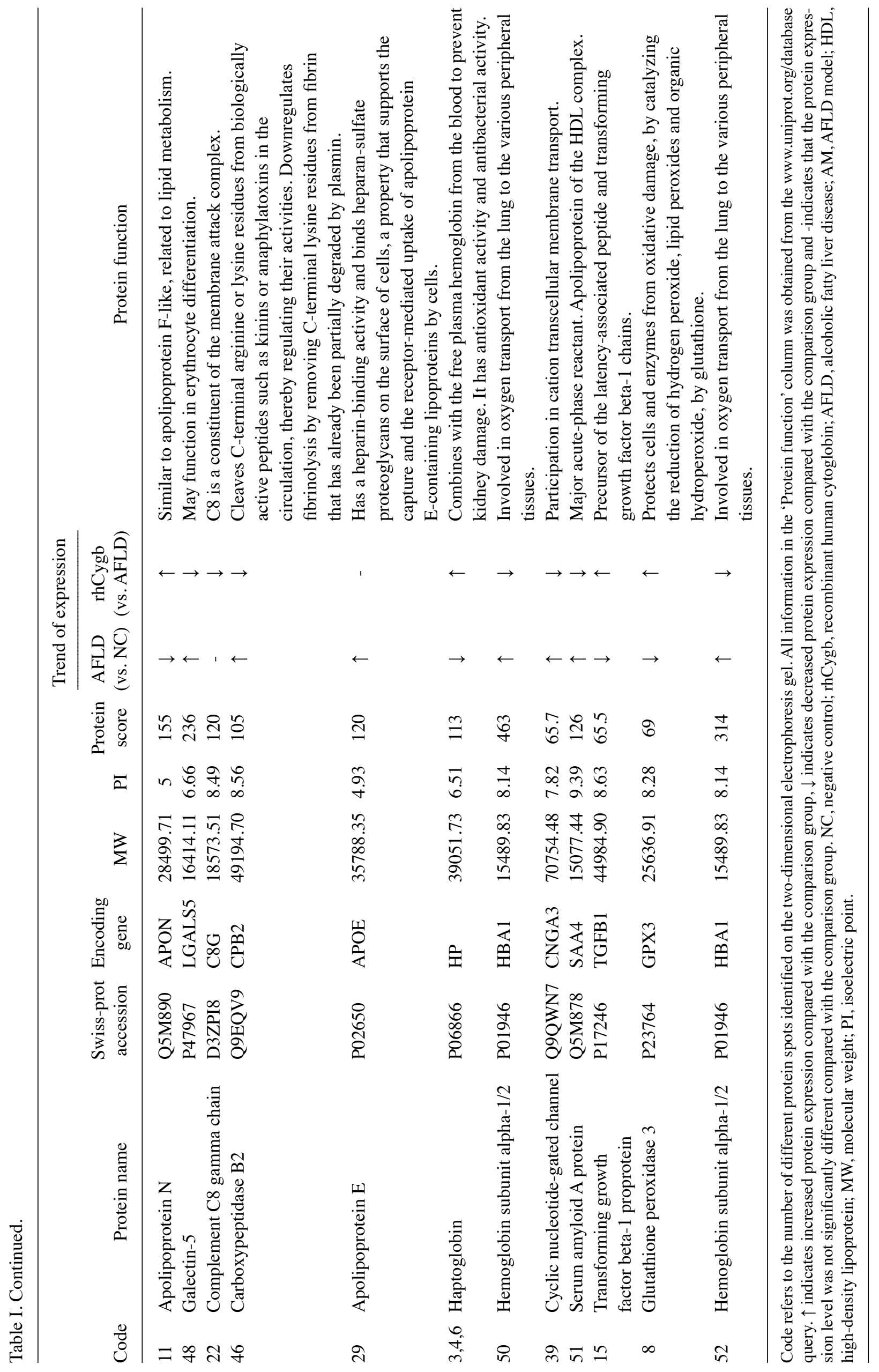




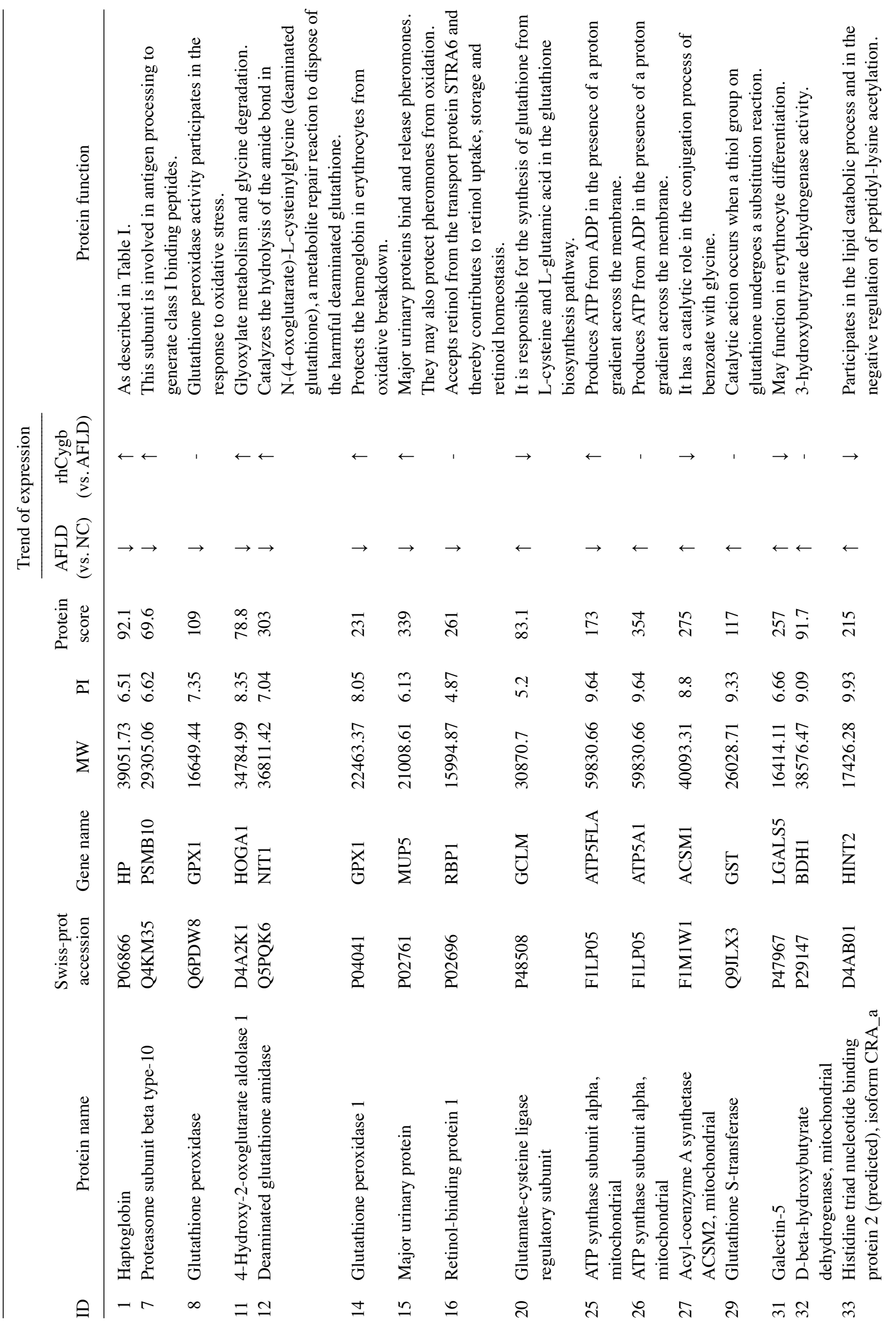


clusters (purple lines are lighter). In addition, purple term clusters represented by monocarboxylic acid biosynthetic process were not associated with other term clusters.

\section{Discussion}

The results of a previous study by our group indicated that rhCygb reduces alcohol-induced liver injury in rats and significantly reverses the AFLD-associated changes in the levels of serum biomarkers; in vitro, rhCygb significantly reduced the proliferation of KCs and TNF- $\alpha$ expression in lipopolysaccharide (LPS)-induced KCs (12). However, the underlying mechanisms of the effects of rhCygb on AFLD have remained to be determined. In the present study, a rat model of AFLD was established and treated with rhCygb. Rat livers and serum were collected from the different treatment groups and the differentially expressed proteins were identified in rat livers and serum using 2-DE and MALDI-TOF-MS.

A total of 53 differentially expressed proteins were observed in the serum samples and the identities of 26 proteins were successfully uncovered using mass spectrometry and the NCBI databases. According to GO categories and Reactome Gene Sets, differentially expressed proteins were primarily involved in binding and uptake of ligands by scavenger receptors, response to corticosteroid, plasma lipoprotein remodeling, regulation of complement cascade, blood circulation, acute inflammatory response and camera-type eye development. The clusters obtained were primarily associated with the regulation of lipoproteins in the blood and immune responses, suggesting that Cygb may serve a therapeutic role by regulating the body's immune response and lipid metabolism.

In addition, 40 differentially expressed proteins were observed in the liver samples and the identities of 20 proteins were successfully determined using mass spectrometry and the NCBI databases. According to the GO categories, differentially expressed proteins were primarily involved in the hydrogen peroxide catabolic process, response to nutrient, response to monosaccharide, monocarboxylic acid biosynthetic process and response to peptide hormone. These five major GO categories belong to cellular metabolic processes, indicating that Cygb may serve its therapeutic role primarily by affecting cell metabolism in the liver. The partially identified proteins are discussed further below.

Haptoglobin acts as an antioxidant and antibacterial agent and is able to remove free plasma hemoglobin to prevent kidney damage (16). In the present study, the expression of haptoglobin in the ALFD group was impaired in the 2-DE analysis of both liver and serum. It is hypothesized that this may have been due to the stimulation of alcohol that resulted in a decrease in the expression of haptoglobin, which in turn reduced its protective effects on the liver. The liver continues to accumulate lesions under other stimuli, eventually developing into AFLD. In the rhCygb group, the expression levels of haptoglobin returned to normal levels and this may underlie the effects of Cygb. In addition to the results of the present study, previous studies reported that Cygb affects the expression of haptoglobin in liver fibrosis (11) and in an atherosclerosis model (17).

The primary function of glutathione peroxidase 3 (Gpx3) is to protect cells and enzymes from oxidative damage by catalyzing the reduction of hydrogen peroxide, lipid peroxides and organic 
Table III. Gene symbols included in each GO term and reactome gene sets of the serum samples.

\begin{tabular}{lll}
\hline GO term & \multicolumn{1}{c}{ Term name } & \multicolumn{1}{c}{ List of genes } \\
\hline R-RNO-2173782 & Binding and uptake of ligands by scavenger receptors & ALB, HP, HBA1, APOE \\
GO:0031960 & Response to corticosteroid & HP, APOA2, TGFB1, GPX3, SULT1A1, CNGA3 \\
R-RNO-8963899 & Plasma lipoprotein remodeling & ALB, APOA2, APOE \\
R-RNO-977606 & Regulation of complement cascade & C4A, CPB2, C8G \\
GO:0008015 & Blood circulation & ALB, HBA1, APOE, TGFB1, SULT1A1 \\
GO:0002526 & Acute inflammatory response & HP, APOA2, SAA4 \\
GO:0043010 & Camera-type eye development & TGFB1, ACTB, CNGA3
\end{tabular}

GO, Gene Ontology; R-RNO, Reactome Gene Sets-Rattus norvegicus; All the define of the gene names were described in Table I; All terms are obtained by gene enrichment in the corresponding 'List of genes'.

Table IV. Gene symbols included in each GO term of the liver samples.

\begin{tabular}{lll}
\hline GO term & \multicolumn{1}{c}{ Term name } & \multicolumn{1}{c}{ List of genes } \\
\hline GO:0042744 & Hydrogen peroxide catabolic process & GPX1, HP, HBA1, GCLM, BDH1 \\
GO:0007584 & Response to nutrient & GPX1, HP, RBP1, GCLM, BDH1 \\
GO:0034284 & Response to monosaccharide & GPX1, HP, GCLM, LOC259246 \\
GO:0072330 & Monocarboxylic acid biosynthetic process & RBP1, HOGA1, ACSM1 \\
GO:0043434 & Response to peptide hormone & HP, BDH1, LOC259246 \\
\hline
\end{tabular}

GO, Gene Ontology; LOC259246, the gene name of the 'Major urinary protein' in Rat genome database; All the define of the gene names were described in Table II; All terms are obtained by gene enrichment in the corresponding 'list of genes'.

hydroperoxides by glutathione $(18,19)$. The expression of Gpx3 was decreased in rats with continuous administration of highly concentrated alcohol, suggesting reduced body antioxidant capacity and damage. Treatment with rhCygb recovered the expression levels of Gpx3 and thus, the body's antioxidant capacity. 2-DE analysis indicated differentially expressed protein levels of glutathione peroxidase 1 (Gpx1), glutamate-cysteine ligase regulatory subunit (Gclm), glutathione S-transferase (GST) and Gpx3, all of which are involved in the metabolism of glutathione. The primary role of Gpx1 is to protect hemoglobin in red blood cells from oxidative damage (20). In the present study, in the liver samples, Gpx1 was downregulated in the AFLD group, whereas in the rhCygb group, the levels were similar to those in the NC group; Gclm expression was increased in the AFLD group and the levels in the rhCygb group were similar to those in the NC group; GST exhibited an increase in expression levels in both the AFLD group and the rhCygb group.

The primary function of serum albumin (Alb) is to regulate the colloid osmotic pressure of blood and it may also inhibit the growth of enteric bacteria (21). In addition, the ability of Alb to inhibit the growth of enteric bacteria may indirectly reduce the activation of LPS-induced KC caused by heavy drinking, after which intestinal permeability is altered and enteric bacteria enter the blood (12). In the AFLD group, the expression of Alb decreased, whereas in the rhCygb group, the expression of Alb increased, suggesting that Cygb may reduce the imbalance of blood colloid osmotic pressure caused by long-term drinking by increasing the expression of Alb.
Psmd1 is a component of the $26 \mathrm{~S}$ proteasome. The $26 \mathrm{~S}$ proteasome maintains protein homeostasis by removing misfolded or damaged proteins that may disrupt cell function (22-25). The protein expression levels of Psmd1 were decreased in the AFLD group and in the serum of the rhCygb group, they were restored to levels similar to those in the NC group. A decrease in the levels of Psmd1 may result in aberrant accumulation of erroneously misfolded proteins, which may impair cell function, thus resulting in a series of pathological changes (22-25).

Proteasome subunit $\beta$ type-5 (Psmb5) is a component of the $20 \mathrm{~S}$ core proteasome complex and is involved in the proteolytic degradation of the majority of intracellular proteins (26). Psmb5 may be associated with two $19 \mathrm{~S}$ regulatory particles to form a $26 \mathrm{~S}$ proteasome. The String database indicates that Psmb5 interacts with Psmd1 (27). In the present study, it was suggested that rhCygb treatment reduced the expression levels of Psmb5.

Serum amyloid A protein (Saa4) is an apolipoprotein of the major acute phase reactants and HDL complexes $(28,29)$. Saa4 is primarily expressed in the liver (30), recruits immune cells to the site of inflammation and transports cholesterol to the liver for secretion into the bile (31). In the present study, the expression of Saa4 in the AFLD group was significantly increased compared with that in the NC group and Saa4 expression in the rhCygb group was similar to the levels observed in the NC group. The change in the expression levels of Saa4 in serum indirectly reflects the therapeutic effect of Cygb. 
A

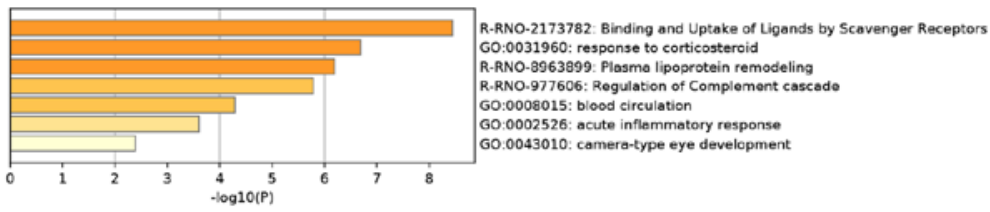

B
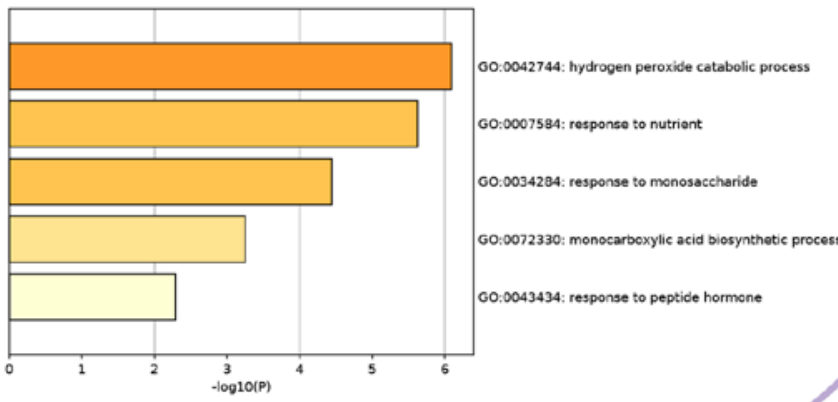

C

Binding and uptake of ligands by scavenger receptors

Response to corticosteroid

Plasma lipoprotein remodeling

Regulation of Complement cascade

Blood circulation

Acute inflammatory response

Camera-type eye development
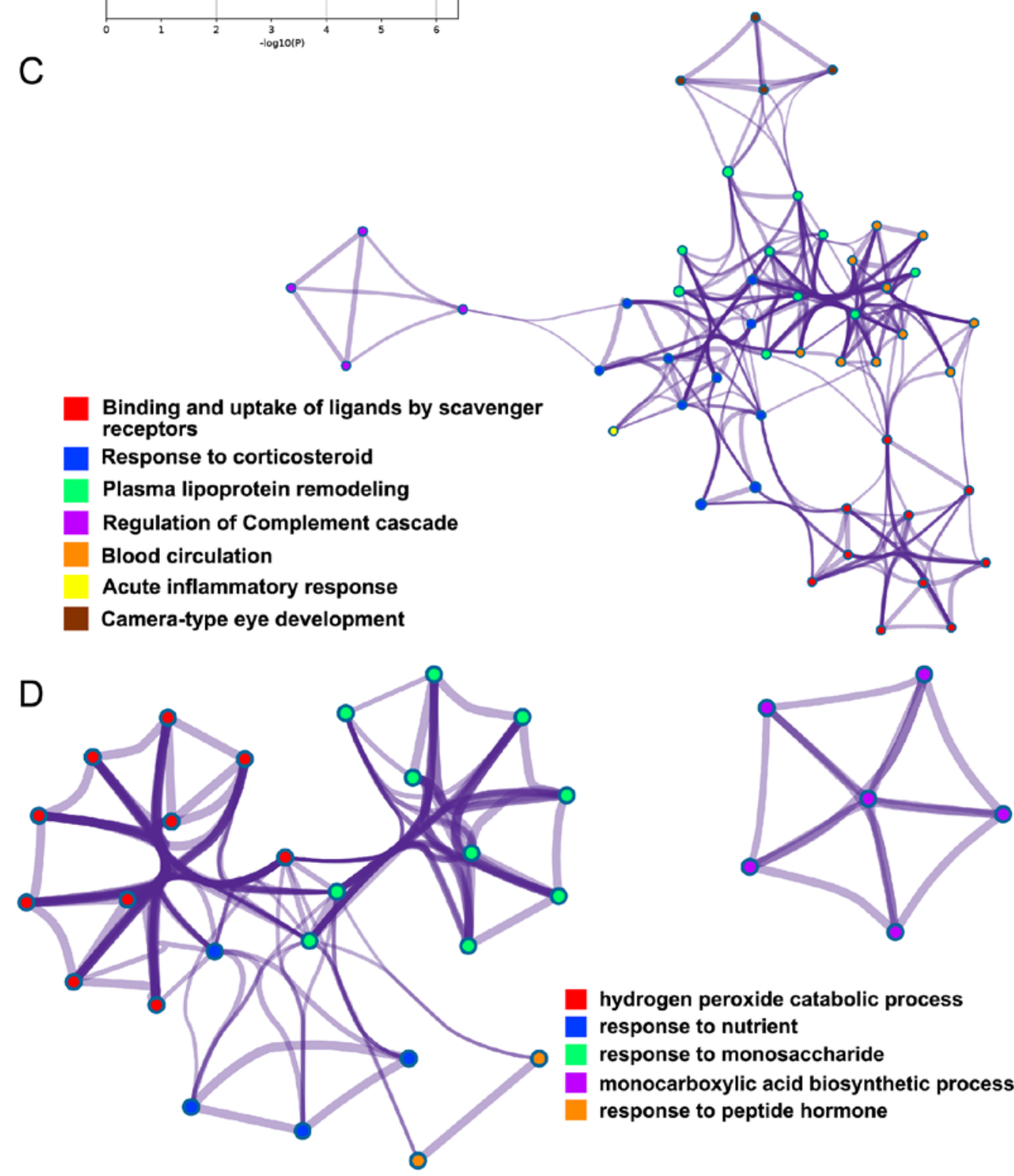

hydrogen peroxide catabolic process

response to nutrient

response to monosaccharide

monocarboxylic acid biosynthetic process

response to peptide hormone

Figure 4. Bioinformatics analysis. (A) Heatmap of serum samples identified by GO analysis. R-RNO-2173782; GO:0031960; R-RNO-8963899; R-RNO-977606; GO:0008015; GO:0002526; GO:0043010. (B) Heatmap of liver samples identified by GO analysis. GO:0042744; GO:0007584; GO:0034284; GO:0072330; GO:0043434. (C) Enriched ontology clusters of serum samples, colored by cluster ID. (D) Enriched ontology clusters of liver samples, colored by cluster ID. GO, Gene Ontology; R-RNO, Reactome Gene Sets-Rattus norvegicus.

Complement $\mathrm{C} 4$ (C4) is a major histocompatibility complex class-III protein, which is essential for the propagation of the classical complement pathway $(32,33)$. Derived from proteolytic degradation of complement $\mathrm{C} 4, \mathrm{C} 4 \mathrm{a}$ anaphylatoxin is a mediator of local inflammatory processes. $\mathrm{C} 4 \mathrm{a}$ induces the contraction of smooth muscle, increases vascular permeability 
and causes histamine release from mast cells and basophilic leukocytes (34). In the present study, the expression of complement $\mathrm{C} 4$ was decreased in the AFLD group and the expression of rhCygb was similar to that in the NC group.

Cygb regulates the concentration of lipoproteins in serum by regulating the expression of apolipoprotein A-II (Apoa2), apolipoprotein $\mathrm{N}$ (Apon) and apolipoprotein $\mathrm{E}$ (Apoe), where Apoa 2 and Apoe are the major apolipoproteins and are involved in the regulation of lipoprotein metabolism (35). The String database indicates that Apon 2 is co-expressed with Apon. The ability of Cygb to regulate lipoprotein in serum was reported in a previous study by our group (12).

To better understand the association between differentially expressed proteins following Cygb treatment, the GO terms were identified and cast into term clusters. For the differentially expressed proteins in the liver, by hierarchically clustering significant terms into the tree, the five term clusters were divided into two parts, of which the purple term clusters represented by the monocarboxylic acid biosynthetic process are separate parts, indicating that the purple term clusters are a separate metabolic process affected by Cygb. The remaining 4 term clusters are linked to each other to form a network of relationships, indicating that the differential proteins of these four term clusters act together on a biological process. Therefore, the effect of Cygb on intracellular metabolism is primarily divided into two parts-one is monocarboxylic acid biosynthetic process (purple term clusters) and the other is primarily related to the reaction of intracellular metabolites, of which red term clusters (hydrogen peroxide catabolic process) are the decomposition of hydrogen peroxide, which is a detoxification mechanism of Cygb.

From the differentially expressed proteins in serum, by hierarchically clustering important terms into a tree, seven term clusters were divided into three parts, where the purple term cluster represented by the adjustment of the complement cascade was the most independent, indicating that the purple term cluster is an independent biological process of Cygb. The remaining six term clusters were joined to each other to form a network of relationships, indicating that the differentially expressed proteins contained in the six terminology clusters act together on biological processes. The blue term cluster representing the response to corticosteroids, the green term cluster representing plasma lipoprotein remodeling and the orange term cluster representing the blood circulation were closely intertwined with each other. The differentially expressed proteins contained in the three terminologies may together form a pathway that is affected by Cygb. Therefore, the influence of Cygb on the biological process of the organism is primarily divided into three parts: i) Regulation of the complement cascade (purple term cluster), ii) the steady state of the blood system (six term clusters other than the purple term cluster) and iii) maintenance of dynamic balance of blood components (blue, green and orange term clusters).

In conclusion, the present study continuing on from the previous study by our group (12), indicated that Cygb exhibited beneficial effects in a rat model of AFLD. To further explore the mechanisms of action of Cygb, 2-DE, MS identification and bioinformatics analysis of differentially expressed proteins was performed. A total of 20 differentially expressed proteins were identified in the liver and all of these were linked to intracellular metabolism, indicating that $\mathrm{Cygb}$ primarily exerts its effects in AFLD by affecting cellular metabolic pathways (such as response to nutrient and monocarboxylic acid biosynthetic process). In particular, Cygb affected the decomposition of hydrogen peroxide to exert a detoxifying effect. In addition, 26 differentially expressed proteins were identified in the serum, which were primarily involved in the body's immune response and regulation of blood components, suggesting that $\mathrm{Cygb}$ may affect biological processes including regulation of immune function and maintenance of the blood component balance (e.g. of scavengers) body-to-ligand binding and uptake, response to corticosteroids and plasma lipoprotein remodeling, to exert its therapeutic effects in AFLD.

\section{Acknowledgements}

Not applicable.

\section{Funding}

This work was supported by a grant from Guangdong Province Science and Technology Plan Project (grant no. 2013A022100027) and Guangzhou Science and Technology Project (grant no. 201804010046).

\section{Availability of data and materials}

The datasets used and/or analyzed during the current study are available from the corresponding author on reasonable request.

\section{Authors' contributions}

WQD and ZL conceived and designed the experiments. ZRZ, JW and BHC performed the experiments and analyzed the data. ZGY and WW analyzed the data and contributed reagents/materials/analysis tools. YMX and ZYW performed the experiments. PW and YMX analyzed the data. WQD, ZL and ZRZ drafted the manuscript. All authors read and approved the final manuscript.

\section{Ethics approval and consent to participate}

All experimental procedures were performed in accordance with the institutional guidelines approved by the Chinese Association of Laboratory Animal Care. All experimental protocols were approved by the IACUC at Southern Medical University (Guangzhou, China).

\section{Patient consent for publication}

Not applicable.

\section{Competing interests}

The authors declare that they have no competing interests.

\section{References}

1. Basra S and Anand BS: Definition, epidemiology and magnitude of alcoholic hepatitis. World J Hepatol 3: 108-113, 2011. 
2. Gaggini M, Morelli M, Buzzigoli E, DeFronzo RA, Bugianesi E and Gastaldelli A: Non-alcoholic fatty liver disease (NAFLD) and its connection with insulin resistance, dyslipidemia, atherosclerosis and coronary heart disease. Nutrients 5: 1544-1560, 2013.

3. Jung UJ and Choi MS: Obesity and its metabolic complications: The role of adipokines and the relationship between obesity, inflammation, insulin resistance, dyslipidemia and nonalcoholic fatty liver disease. Int J Mol Sci 15: 6184-6223, 2014.

4. Fon Tacer K and Rozman D: Nonalcoholic fatty liver disease: Focus on lipoprotein and lipid deregulation. J Lipids 2011: 783976, 2011.

5. Suk KT, Kim MY and Baik SK: Alcoholic liver disease: Treatment. World J Gastroenterol 20: 12934-12944, 2014.

6. Kawada N, Kristensen DB, Asahina K, Nakatani K, Minamiyama Y, Seki S and Yoshizato K: Characterization of a stellate cell activation-associated protein (STAP) with peroxidase activity found in rat hepatic stellate cells. J Biol Chem 276 : 25318-25323, 2001.

7. Burmester T, Ebner B, Weich B and Hankeln T: Cytoglobin: A novel globin type ubiquitously expressed in vertebrate tissues. Mol Biol Evol 19: 416-421, 2002.

8. Yoshizato K, Thuy le TT, Shiota G and Kawada N: Discovery of cytoglobin and its roles in physiology and pathology of hepatic stellate cells. Proc Jpn Acad Ser B Phys Biol Sci 92: 77-97, 2016

9. Fordel E, Thijs L, Moens L and Dewilde S: Neuroglobin and cytoglobin expression in mice. Evidence for a correlation with reactive oxygen species scavenging. FEBS J 274: 1312-1317, 2007.

10. Gardner AM, Cook MR and Gardner PR: Nitric-oxide dioxygenase function of human cytoglobin with cellular reductants and in rat hepatocytes. J Biol Chem 285: 23850-23857, 2010.

11. Li Z, Wei W, Chen B, Cai G, Li X, Wang P, Tang J and Dong W: The effect of rhCygb on CCl4-induced hepatic fibrogenesis in rat Sci Rep 6: 23508, 2016

12. Wen J, Wu Y, Wei W, Li Z, Wang P, Zhu S and Dong W: Protective effects of recombinant human cytoglobin against chronic alcohol-induced liver disease in vivo and in vitro. Sci Rep 7: 41647, 2017.

13. Wang Y, Liang X, Yang J, Wang H, Tan D, Chen S, Cheng J, Chen Y, Sun J, Rong F, et al: Improved performance of quantitative collagen parameters versus standard histology in longitudinal assessment of nonadvanced liver fibrosis for chronic hepatitis B. J Viral Hepat 25: 598-607, 2018.

14. Zhou J, Li J, Yu Y, Liu Y, Li H, Liu Y, Wang J, Zhang L, Lu X, Chen $Z$ and Zuo D: Mannan-binding lectin deficiency exacerbates sterile liver injury in mice through enhancing hepatic neutrophil recruitment. J Leukoc Biol 105: 177-186, 2019.

15. He WQ, Chen XJ, Wen YQ, Li YZ, He H and Chen Q: Detection of hepatitis B virus-like nucleotide sequences in liver samples from murine rodents and asian house shrews. Vector Borne Zoonotic Dis 19: 781-783, 2019.

16. Fagoonee S, Gburek J, Hirsch E, Marro S, Moestrup SK, Laurberg JM, Christensen EI, Silengo L, Altruda F and Tolosano E: Plasma protein haptoglobin modulates renal iron loading. Am J Pathol 166: 973-983, 2005.

17. Ou L, Li X, Chen B, Ge Z, Zhang J, Zhang Y, Cai G, Li Z, Wang $\mathrm{P}$ and Dong W: Recombinant human cytoglobin prevents atherosclerosis by regulating lipid metabolism and oxidative stress. J Cardiovasc Pharmacol Ther 23: 162-173, 2018.
18. Qi X, Ng KT, Lian QZ, Liu XB, Li CX, Geng W, Ling CC Ma YY, Yeung WH, Tu WW, et al: Clinical significance and therapeutic value of glutathione peroxidase 3 (GPx3) in hepatocellular carcinoma. Oncotarget 5: 11103-11120, 2014.

19. Arthur JR: The glutathione peroxidases. Cell Mol Life Sci 57: $1825-1835,2000$

20. Lubos E, Loscalzo J and Handy DE: Glutathione peroxidase-1 in health and disease: From molecular mechanisms to therapeutic opportunities. Antioxid Redox Signal 15: 1957-1997, 2011

21. Dich J, Hansen SE and Thieden HI: Effect of albumin concentration and colloid osmotic pressure on albumin synthesis in the perfused rat liver. Acta Physiol Scand 89: 352-358, 1973.

22. Rock KL, Gramm C, Rothstein L, Clark K, Stein R, Dick L, Hwang D and Goldberg AL: Inhibitors of the proteasome block the degradation of most cell proteins and the generation of peptides presented on MHC class I molecules. Cell 78: 761-771, 1994.

23. Chen B, Retzlaff M, Roos T and Frydman J: Cellular strategies of protein quality control. Cold Spring Harb Perspect Biol 3: a004374, 2011.

24. Voges D, Zwickl P and Baumeister W: The 26S proteasome: A molecular machine designed for controlled proteolysis. Annu Rev Biochem 68: 1015-1068, 1999.

25. Rodriguez KA, Edrey YH, Osmulski P, Gaczynska M and Buffenstein R: Altered composition of liver proteasome assemblies contributes to enhanced proteasome activity in the exceptionally long-lived naked mole-rat. PLoS One 7: e35890, 2012.

26. Fujito NT and Nonaka M: Highly divergent dimorphic alleles of the proteasome subunit beta type-8 (PSMB8) gene of the bichir Polypterus senegalus: Implication for evolution of the PSMB8 gene of jawed vertebrates. Immunogenetics 64: 447-453, 2012.

27. da Fonseca PC, He J and Morris EP: Molecular model of the human 26S proteasome. Mol Cell 46: 54-66, 2012.

28. Wang L and Colón W: The interaction between apolipoprotein serum amyloid A and high-density lipoprotein. Biochem Biophys Res Commun 317: 157-161, 2004

29. de Beer MC, Yuan T, Kindy MS, Asztalos BF, Roheim PS and de Beer FC: Characterization of constitutive human serum amyloid A protein (SAA4) as an apolipoprotein. J Lipid Res 36: 526-534, 1995.

30. Uhlar CM and Whitehead AS: Serum amyloid A, the major vertebrate acute-phase reactant. Eur J Biochem 265: 501-523, 1999.

31. Eklund KK, Niemi K and Kovanen PT: Immune functions of serum amyloid A. Crit Rev Immunol 32: 335-348, 2012.

32. Mayilyan KR: Complement genetics, deficiencies, and disease associations. Protein Cell 3: 487-496, 2012.

33. Birmingham DJ and Hebert LA: The complement system in lupus nephritis. Semin Nephrol 35: 444-454, 2015.

34. Hugli TE, Marceau F and Lundberg C: Effects of complement fragments on pulmonary and vascular smooth muscle. Am Rev Respir Dis 135: S9-S13, 1987.

35. Mahley RW, Innerarity TL, Rall SC Jr and Weisgraber KH: Plasma lipoproteins: Apolipoprotein structure and function. J Lipid Res 25: 1277-1294, 1984.

This work is licensed under a Creative Commons Attribution-NonCommercial-NoDerivatives 4.0 International (CC BY-NC-ND 4.0) License. 